\title{
Dissecting estimation of conductances in subthreshold regimes*
}

\author{
Catalina Vich ${ }^{\dagger 1}$ and Antoni Guillamon ${ }^{\ddagger 2}$ \\ ${ }^{1}$ Dept. of Mathematics and Computer Science - Escola Politècnica \\ Superior, Universitat de les Illes Balears, Anselm Turmeda, Ctra. \\ de Valldemossa, km. 7,5, 07122 Palma \\ ${ }^{2}$ Department of Mathematics - EPSEB, Universitat Politècnica de \\ Catalunya, Avda. Dr. Marañón, 44-50, 08028 Barcelona,
}

September 15th, 2015

\begin{abstract}
We study the influence of subthreshold activity in the estimation of synaptic conductances. It is known that differences between actual conductances and the estimated ones using linear regression methods can be huge in spiking regimes, so caution has been taken to remove spiking activity from experimental data before proceeding to linear estimation. However, not much attention has been paid to the influence of ionic currents active in the non-spiking regime where such linear methods are still profusely used. In this paper, we use conductance-based models to test this influence using several representative mechanisms to induce ionic subthreshold activity. In all the cases, we show that the currents activated during subthreshold activity can lead to significant errors when estimating synaptic conductance linearly. Thus, our results add a new warning message when extracting conductance traces from intracellular recordings and the conclusions concerning neuronal activity that can be drawn from them. Additionally, we present, as a proof of concept, an alternative method that takes into account the main nonlinear effects of specific ionic subthreshold currents. This method, based on the quadratization of the subthreshold dynamics, allows us to reduce the relative errors of the estimated conductances by more than one order of magnitude. In experimental conditions, under appropriate fitting to canonical models, it could be useful to obtain better estimations as well even under the presence of noise.

Keywords: Synaptic conductance estimation, Conductance-based model, Subthreshold activity, Quadratization, Intracellular recordings.
\end{abstract}

\footnotetext{
* Article accepted in Journal of Computational Neuroscience

†catalina.vich@uib.es

$\ddagger$ antoni.guillamon@upc.edu
} 


\section{Introduction}

One of the most challenging problems in neuroscience is to unveil brain's connectivity, which may vary depending on the task being performed. In order to solve this riddle, to infer this connectivity and to understand the dynamics of information, processing methods are sought both from experimental and theoretical perspective.

A "local" simplified situation, despite of its global repercussion, is trying to find out which signal is receiving a single neuron subjected to a bombardment of synaptic inputs and then discern the temporal contributions of global excitation from those of global inhibition. This quantitative information is important for the integrative properties of cortical neurons which are believed to be altered under high-conductance states, see for instance Destexhe et al (2003). The relationship between the modulation of excitatory and inhibitory time courses is also important to get information about the wiring architecture of the cortex since it may help to distinguish between phase insensitive cortical coupling or spatial phase selective coupling, see McLaughlin et al (2000). On the other hand, this joint information is useful to study both the balance and the concurrence of excitation and inhibition, which are crucial features in many neuronal problems, see Wehr and Zador (2003) and Lombardi et al (2012) among others. Moreover, disruption of these features leads to severe disorders, see references in Berg and Ditlevsen (2013), so it is relevant to obtain precise estimations of the activity arriving to a specific cell.

Due to the multitude and the variety of synaptic contacts, obtaining direct measurements of the synaptic currents that the neuron is receiving at each moment in time is something unreachable. Therefore, inverse methods appear as an alternative to estimate the input (mainly, the conductances) from experimental measures. Experiments that provide membrane potential time courses from intracellular recordings of cortical cells are relevant in this regard, and have been carried out for different cell types in different brain areas, see Hirsch et al (1998), Anderson et al (2000), Wehr and Zador (2003) and Monier et al (2008).

Theoretical contributions have been mostly focused on the approach with Fokker-Planck equations to derive the mean and the variance of the whole temporal course for both the excitatory and the inhibitory inputs, see for instance Rudolph et al (2004). However, the main shortcoming, both of many experimental papers and the abovementioned theoretical methods, is the need for recording several membrane potential time courses assuming invariance of the conductances time courses across them. To overcome such problems, the FokkerPlanck approach has been refined to avoid double recordings by using maximum likelihood estimators, see Pospischil et al (2009). Recently, new efforts have been devoted to obtain direct estimations of the excitatory and inhibitory conductances: Bédard et al (2011) takes advantage of oversampling the membrane potential with respect to the conductances time-scale, whereas other authors, see Kobayashi et al (2011), Paninski et al (2012), Berg and Ditlevsen (2013), Lankarany et al (2013a), Lankarany et al (2013b) and Closas (2014), take ad- 
vantage statistical inference methods to extract on-line activity.

In this paper, we will be devoted to multi-trial in sillico experiments, but the main messages can be also exported to on-line strategies since they focus on the linear versus nonlinear character of the inferring methods. In fact, many experimental and theoretical methods rely on the fact that the neuron acts as a linear filter, and caution has to be taken to avoid estimations in spiking regimes, see Guillamon et al (2006). Some strategies trying to linearise the $I_{a p p}-v$ relationship, see for instance Anderson et al (2000) and Wehr and Zador (2003), have proven not to be valid, as pointed out in Guillamon et al (2006) where computational models were used to show that nonlinear $I_{a p p}-v$ relationships cannot be eliminated through standard filtering, and thus linear estimations are not reliable (errors can be of the order of 800\%). Unfortunately, conclusions are still drawn from experimental studies by means of this type of estimations, see for instance Bennett et al (2013).

However, given that the basic reason for the misestimations is that some nonlinear terms are active, the problem could be spread also over non-spiking regimes as well due to the eventual activity of subthreshold ionic channels, widely described from the eighties after seminal works as Hotson and Prince (1980). This nonlinear subthreshold activity cannot be discarded even in the most careful experimental results on conductance estimation obtained up-todate, see for instance Figure 6 in Rudolph et al (2004) where this type of channels is explicitly considered. In that paper, Rudolph et al. already warned about errors caused in the estimations attributed to the activation of subthreshold voltage-dependent membrane conductances but they concluded that these conductances did not seem to have strong effects on the estimates. Of course, pharmacological blocks can reduce the activity of some targeted channels, but still it is actually difficult to completely reduce the neuron's activity to a pure passive filter.

In this paper, we aim at showing that misestimations induced by the presence of subthreshold-activated ionic currents are ubiquitous and independent on the mechanisms that activate these currents. For this purpose, firstly, we take a conductance-based computational model of a spiking neuron with two significant types of subthreshold currents, a calcium-activaded potassium afterhyperpolarizing current (AHP) and a low-threshold calcium current (LTS); secondly, to elude the possible contaminating effects (specially on the AHP currents) of the spiking activity, we take a conductance-based model of a non-spiking neuron which currents are a persistent sodium current $(\mathrm{NaP})$ and an h-current. Both models are fed with realistic excitatory and inhibitory conductance traces obtained from an in sillico (noisy) network of visual cortex (see McLaughlin et al (2000) and Tao et al (2004)). The resulting voltage traces are then used to obtain estimated conductance courses by linear estimation methods. Finally, the input synaptic drive and the estimated conductance courses are compared in order to quantify and analyse the estimation errors due to the presence of the above mentioned subthreshold currents. We also study alternative procedures to estimate the conductances taking into account the nonlinear effects. On one hand, we explore the role of ionic channels time-scales and, on the other hand, 
we propose a method based on a quadratization of the subthreshold dynamics. In Section 2, we firstly provide the neuron models together with the input synaptic drive. For the pyramidal cell model, we construct an index to discern whether each subthreshold current is dominant over all the other ionic currents whereas, for the stellate cell model, we refer to the quadratization procedure described in Rotstein (2015) as a simplification of the model. Then, in this same section, we describe the linear filtering method and we provide two alternative methods to estimate conductances in the subthreshold regime. Section 3 is devoted to the results which imply that caution has to be applied also in subthreshold regimes to ensure the absence of nonlinear behaviours. In particular, attention must be paid to check that $\mathrm{Ca}^{2+}$-dependent $\mathrm{K}^{+}$currents and other ionic currents responsible for subthreshold oscillations are inactive before proceeding to linearly estimate the synaptic conductances from voltage traces. We also analyse how the alternative procedures improve the linear regression.

\section{Methods}

As we have mentioned in Section 1, two different conductance-based models are considered: a first one where a subthreshold current and a hyperpolarized current coexist, and a second one with two currents that jointly induce subthreshold oscillations. In this section, we present these two models and we also provide two different approaches to estimate conductances which are going to be tested in the Section 3 .

\subsection{Models and data treatment}

\subsubsection{Pyramidal cell model with an AHP and an LTS currents}

The first model we consider in our simulations is a simplification of a model for a pyramidal neuron given in Wang (1998) adding a low-threshold $\mathrm{Ca}^{2+}$ current $I_{L T S}$ given in Destexhe et al (1993); its main terms are expressed in the equation:

$$
C_{m} \frac{d V}{d t}=-I_{L}-I_{i o n}+I_{a p p}-I_{s y n},
$$

where $C_{m}$ is the capacitance, $I_{i o n}=I_{N a}+I_{K}+I_{C a}+I_{A H P}+I_{L T S}$ the ionic current, $I_{s y n}$ the synaptic current, $I_{a p p}$ the applied current and $I_{L}$ the leak. The model is chosen to have the minimal complexity to analyse the problem, with a spiking mechanism provided by sodium and potassium currents, $I_{N a}, I_{K}$, and two different current sources for subthreshold activity: a calcium-activated afterhyperpolarising potassium current, $I_{A H P}$, and a low-threshold activating channel, $I_{L T S}$. These two currents are chosen to display different ways to induce ionic activity in subthreshold regimes. On one side, the $A H P$ is generated by slow currents that turn on right after the spike; on the other hand, low-threshold currents are usually activated at voltage values above resting potential but not high enough to evoke spikes. 
The $I_{\{N a, K, C a, A H P, L T S\}}$ terms model the respective ionic currents. In particular, we stress that AHP is a potassium current given by $I_{A H P}=g_{A H P} c /(c+$ $\left.K_{D}\right)\left(v-V_{K}\right)$, mediated by the concentration of calcium $c:=\left[\mathrm{Ca}^{2+}\right]$, which has its own dynamics mainly dependent on $I_{C a}$ and a large time constant $\tau_{C a}$. On the other hand, the $L T S$ current is given by $I_{L T S}=g_{L T S} m_{L T S, \infty}^{3} h_{L T S}(v-$ $\left.V_{C a}\right)$. The function $h_{L T S}$ is a sigmoidal function with a low inflection point that induces the desired low-threshold activation. Details on the rest of equations and parameter values of the model can be found in Appendix A.

Index of dominance of subthreshold currents. In order to have, for this first example, a clear description of the time intervals where the currents $I_{A H P}$ and $I_{L T S}$ prevail over the rest of the currents, we have defined an index $\chi(t)$ as

$$
\chi(t)=\frac{-I_{A H P}(t)-I_{L T S}(t)}{\sqrt{I_{\text {ion }}(t)^{2}+\left(I_{A H P}(t)+I_{L T S}(t)\right)^{2}}},
$$

where $I_{\text {ion }}(t)=I_{N a}(t)+I_{K}(t)+I_{C a}(t)$.

Note that, because of the respective reversal potentials, when the index $\chi(t)$ is greater than $\sqrt{2} / 2,-I_{L T S}$, which is positive, is the dominant current whereas the index $\chi(t)$ being smaller than $-\sqrt{2} / 2$ implied that the dominant current is $-I_{A H P}$, which is negative. Otherwise, the neuron is spiking and so the other ionic currents prevail over the sum of $I_{L T S}$ and $I_{A H P}$. We also point out that the index is not defined when $I_{i o n}(t)^{2}+\left(I_{A H P}(t)+I_{L T S}(t)\right)^{2}=0$; we have included a condition in the code so as to maintain the value of $\chi(t-\Delta t)$ when $I_{\text {ion }}(t)^{2}+\left(I_{A H P}(t)+I_{L T S}(t)\right)^{2}<T O L$, with $T O L=10^{-12}$. However, this only occurs transiently and does not affect any result in this paper.

\subsubsection{Stellate cell model with NaP- and h- currents}

The second model we consider in our simulations is a reduced model for medial entorhinal cortex stellate cell given in Rotstein et al (2006) that displays subthreshold oscillations. The only considered activated currents in the model are the persistent sodium $I_{N a P}$ current and the h- $\left(I_{h}\right)$ current, which are involved in rhythmic subthreshold oscillations (see Dickson et al (2000), for instance). The main terms of this model also follow an equation of type (1) but here the ionic current term is $I_{i o n}=I_{N a P}+I_{h}$. The dynamics of the persistent sodium current evolves in a fast time scale for what we can suppose that its gating variable is at the steady-state. Moreover, even though h-currents usually have two components (the fast and the slow one), in this model we only consider the fast component. Spiking currents are not taken into account to ensure that only subthreshold activity exists. Details on the equations and parameters can be found in Appendix B.

Quadratization. Under the presence of both $I_{N a P}$ and $I_{h}$ currents, since $I_{h}$ is a resonant current and $I_{N a P}$ an amplifying one, the interaction between them 
often induces nonlinearities of quadratic type in the voltage response (see Rotstein (2015)). Since these nonlinear effects cannot be captured by a linearization of the model, a quadratization has been provided in Rotstein (2015) to capture the parabolic shape of the voltage nullcline. The general quadratization is given by

$$
\begin{aligned}
& \frac{d v}{d t}=a v^{2}-w+I(t), \\
& \frac{d w}{d t}=\varepsilon(\alpha v-\lambda-w),
\end{aligned}
$$

where $I(t)=I_{a p p}+I_{s y n}(t)$ and $w$ stands for the set of gating variables. Parameters $a, \alpha, \varepsilon$ and $\lambda$ are considered as constants defined in terms of the biophysical parameters of the original model which capture the geometry of the phase-plane. In this sense, $a$ controls the curvature of the $v$-nullcline, $\alpha$ controls the slope of the $w$-nullcline, $\varepsilon$ stands for the time scale separation between $v$ and $w$, which tends to be small, and $\lambda$ controls the relative displacement between the two nullclines (the $v$ one and the $w$ one).

For the biophysical parameters considered in our model (see Appendix B), $w$ stands for $r_{f}$ and the constant parameters of the quadratization result to be $a=0.1, \alpha=0.4, \varepsilon=0.01$ and $\lambda=-0.2$.

\subsubsection{Synaptic drive}

We assume that the target neuron is stimulated both through the synaptic input $I_{s y n}$ and the applied current $I_{a p p}$. The synaptic input takes the form $I_{s y n}=g_{E}\left(v-V_{E}\right)+g_{I}\left(v-V_{I}\right)$, where $g_{E}=g_{E}(t)$ and $g_{I}=g_{I}(t)$ are prescribed synaptic conductances. As test conductance courses, we will use conductance traces (with a $1 \mathrm{~ms}$ resolution) obtained from a computational network that models layer $4 \mathrm{C} \alpha$ of primary visual cortex, see McLaughlin et al (2000) and Tao et al (2004). The complete conductance traces fed into the pyramidal cell model are shown in Supplementary Figure 1. In the stellate cell model, we have rescaled these data by a factor of 3 in order to adjust to the amplitude of the input used in Rotstein (2015).

\subsubsection{Numerical methods}

The systems of differential equations of both models were integrated using the Runge-Kutta 4-5 method with a fixed time step of $0.05 \mathrm{~ms}$. First, we tested that voltage traces did not change when using lower time steps and higher order variable step methods, but the fact of needing equispaced values for the filtering process was a key point for the method's choice.

Moreover, to solve the integral in Section 3.2.1, we have used the trapezoidal rule with the same time step than for the integration method, $0.05 \mathrm{~ms}$. 


\subsection{Estimation procedures}

\subsubsection{Linear estimation approach}

As we mentioned in the Introduction, some experimental studies try to get rid of spikes and linearise the $I_{a p p}-v$ relationship by filtering the intracellular spiking voltage. Our aim is to mimic these standard experimental procedures and analyse their pitfalls. Thus, as in Guillamon et al (2006), we smooth the membrane potential traces $v(t)$ for a fixed $I_{a p p}$ using a median filter and obtaining a new signal $v_{f i l t}\left(t ; I_{a p p}\right)$. In particular, for each point $p:=(t, v(t))$ of the voltage trace, we compute the median of the values in window which includes $2 N+1$ points and is centered at $p$ :

$$
v_{f i l t}\left(t ; I_{a p p}\right)=\operatorname{median}_{j=-N}^{N}\{v(t+j h)\},
$$

where $h$ is the integration step (that is, $1 / h$ is the sampling frequency in $K H z$ ). In our computations, we have taken $N=10$. We have also explored the possibility that a repetitive application of the same filter could lead to a better smoothing and thus a better approximation using linear methods. However, we have proved that the median filter with an usual recording step does not improve beyond a second successive filtering. Therefore, after this filtering process we get $v_{f i l t}\left(t ; I_{a p p}\right)$ for any time value $t$ and any applied current value $I_{a p p}$.

Then, for each $t$ independently, we estimate the conductances on the basis of linear regression assuming that the solutions of the neuron model are close to the steady-state which implies that the activity of the ionic channels is not significant and, in addition, $v_{f i l t} \approx 0$. We thus estimate the total synaptic conductance $g_{\text {syn }}(t)$ and the effective reversal potential $V_{\text {eff }}(t)$ through

$$
v_{f i l t}\left(t ; I_{a p p}\right)=V_{e f f}(t)+\frac{I_{a p p}}{g_{s y n}(t)},
$$

where $g_{\text {syn }}(t)=g_{E}(t)+g_{I}(t)+g_{L}$ and $V_{\text {eff }}(t)=\left(g_{E}(t) V_{E}+g_{I}(t) V_{I}+g_{L} V_{L}\right) / g_{\text {syn }}(t)$, by presenting $M$ different values of $I_{a p p}$ for each time $t$, where $M(\geq 2)$ is the number of trials each of them with a different value of $I_{a p p}$.

Once we have estimated $g_{s y n}(t)$ and $V_{\text {eff }}(t)$, using $(4)$, we can estimate $g_{E}(t)$ and $g_{I}(t)$ assuming that we know the rest of parameters (namely, $g_{L}, V_{L}, V_{E}$ and $V_{I}$ ) by solving, for each value of $t$, the linear system:

$$
\left\{\begin{array}{l}
g_{E}(t)+g_{I}(t)=g_{s y n}(t)-g_{L}, \\
g_{E}(t) V_{E}+g_{I}(t) V_{I}=g_{s y n}(t) V_{e f f}(t)-g_{L} V_{L} .
\end{array}\right.
$$

\subsubsection{Linearization of the subthreshold ionic currents approach}

The linear estimation is based on the fact that the activity of the ionic channels is not significant, and so the ionic currents are null. However, these currents are activated on the subthreshold regime independently of the spikes. Its time scale changes according to the steady state value of the current and so, a better estimation could be done if we could assume that the ionic channels are in 
the steady state. Therefore, in the phase where the subthreshold currents are dominating and the spiking currents are negligible, the $I-v$ relationship would become

$$
v\left(t ; I_{a p p}\right)=V_{\text {eff }}(t)-\frac{I_{\infty}(t)}{g_{s y n}(t)}+\frac{I_{a p p}}{g_{s y n}(t)},
$$

where $I_{\infty}(t):=I_{\text {ion }, \infty}\left(v\left(t ; I_{\text {app }}\right)\right)$ and $I_{i o n, \infty}(v)$ is the sum of the ionic currents at the steady state. Thus, obtaining a relationship $v=v\left(I_{a p p} ; V_{e f f}, g_{s y n}\right)$ from the implicit equation $v=V_{\text {eff }}-I_{\infty}(v) / g_{\text {syn }}+I_{a p p} / g_{\text {syn }}$, where $V_{\text {eff }}$ and $g_{\text {syn }}$ are thought of as parameters, would allow to have a general formula to estimate $V_{\text {eff }}(t)$ and $g_{\text {syn }}(t)$ for each $t$. Unfortunately, this is not easy to perform but, considering the pyramidal cell model described in section 2.1.1, an interesting observation is that, in the phase where $I_{L T S}$ is dominating $(v \in[-75,-60] \mathrm{mv}$ approximately), the function

$$
I_{L T S, \infty}(v):=g_{L T S} m_{L T S, \infty}(v)^{3} h_{L T S, \infty}(v)\left(v-V_{C a}\right)
$$

can be very well fitted by a straight line. In other words, the range of voltage values where $I_{L T S}$ activates and the other ionic currents are negligible coincides with a straight ramp of the bell-shaped $I_{L T S, \infty}$ function. This observation provides a new approach consisting of approximating $I_{L T S}(v) \approx \alpha_{L T S} v+\beta_{L T S}$, and then, for each $t$, applying the following steps:

1. Obtain the slope $a$ and the intercept $b$ from a linear regression of the set of points $\left\{\left(I_{a p p, j}, v\left(t ; I_{a p p, j}\right)\right)\right\}_{j=1}^{M}$.

2. Estimate $g_{\text {syn }}(t)=1 / a-\alpha_{L T S}$ and $V_{\text {eff }}(t)=\left(b+a \beta_{L T S}\right) /\left(1-a \alpha_{L T S}\right)$.

3. Estimate $g_{E}(t)$ and $g_{I}(t)$ from equation (5).

Observe that taking $\alpha_{L T S}=\beta_{L T S}=0$, we obtain again (4).

\subsubsection{Quadratization approach}

As we have mentioned in Section 2.1.2, when resonant and amplifying currents coexist (see Appendix B for the complete model), it has been proved (see Rotstein (2015)) that the system presents nonlinearities of quadratic type in the voltage response. In turn, it is possible to approximate the model by a minimal model with linear and quadratic terms; this process is also konwn as the quadratization of the original system. More precisely, the quadratization is given by the differential system (3). We reparameterize the system by the slow time $\tau=t \varepsilon$ thus obtaining

$$
\begin{aligned}
& \varepsilon \frac{d v}{d \tau}=a v^{2}(\tau)-w(\tau)+I(\tau) \\
& \frac{d w}{d \tau}=(\alpha v(\tau)-\lambda-w(\tau)) .
\end{aligned}
$$

Since the voltage is a fast variable of system (7) and the gating variable $w$ is slow, the differential system (7) can be considered as a slow-fast system where the difference on the time scales of both variables is given by the parameter $\varepsilon$. 
When $\varepsilon$ tends to zero, the associated system is known as the slow subsystem and contains the singular dynamics of the system (7). Fenichel's geometric theory (see Fenichel (1979)) ensures the persistence of the critical manifold of the slow subsystem when it is perturbed. Therefore, in order to make an estimation of the total current, one can assume the limiting case $\varepsilon=0$ to obtain an approximated expression of the total current. In this case, the second equation of the system is a linear non-autonomous ordinary differential equation which can be solved as

$$
w(\tau)=e^{-\tau}\left(w\left(\tau_{0}\right) e^{\tau_{0}}+\int_{t_{0}}^{\tau}(\alpha v(s)-\lambda) d s\right) .
$$

Moreover, from the first equation of the slow subsystem and the fact that $I(\tau)=$ $I_{\text {syn }}(\tau)+I_{\text {app }}$, we can reconstruct the total input current from

$$
\begin{aligned}
I_{s y n}(\tau)= & -a v^{2}(\tau)+ \\
& e^{-\tau}\left(w\left(\tau_{0}\right) e^{\tau_{0}}+\int_{t_{0}}^{\tau}(\alpha v(s)-\lambda) d s\right)-I_{a p p} .
\end{aligned}
$$

To extract the excitatory and the inhibitory conductances, we can take, for instance, two different injected currents, $I_{a p p, j}, j=1,2$, obtain the respective $I_{\text {syn }, j}(t), j=1,2$, from (8), and finally solve

$$
\left\{\begin{array}{l}
I_{s y n, 1}(t)=-g_{E}(t)\left(v_{1}(t)-V_{E}\right)-g_{I}(t)\left(v_{1}(t)-v_{I}\right), \\
I_{s y n, 2}(t)=-g_{E}(t)\left(v_{2}(t)-V_{E}\right)-g_{I}(t)\left(v_{2}(t)-v_{I}\right) .
\end{array}\right.
$$

It is worth noting that in order to apply (8) one has to make a guess of the initial condition $w\left(\tau_{0}\right)$ which is not observable as it is $v\left(\tau_{0}\right)$. However, the method is robust enough to converge with a wide range of initial conditions.

\section{Results}

The study made in Guillamon et al (2006) showed the goodness of the linear estimation when the system is only driven by the synaptic activity, a regime where the equation (4) holds true. However, it was also shown that the estimations fail when the neuron is either spiking or near to spikes in which case the linear relation between the membrane potential $v_{f i l t}$ and the applied current $I_{a p p}$ is broken. Here, we explore the influence of subthreshold ionic activity in the estimation of synaptic conductances, a paradigm that was not taken into account in that previous work.

The way we proceed is, first, consider the pyramidal cell model described in Section 2.1.1 to study the possible errors caused by an afterhyperpolarization current, $I_{A H P}$, and a subthreshold-activated current, $I_{L T S}$, both together and separately. For this purpose and also to avoid the influence of the currents promoting spikes, we have introduced the $\chi$ index defined in (2) to discriminate the time intervals when either $I_{A H P}, I_{L T S}$ or the spiking currents dominate (see also Figure 1). In the first two regimes we detect important relative errors in estimating the synaptic conductances. For the $I_{L T S}$-dominated regime, we 
have come up with an alternative way to improve the linear estimation based on the method explained in Section 2.2.2. Even though this example already illustrates the misestimations in subthreshold regimes, it could be argued, in the case $I_{A H P}$-dominated regimes, that these misestimations are an artifact of the misestimations in the spiking regime. To enhance our warning message on subthreshold misestimations we have also considered a second model with no spiking mechanisms and two different subthreshold-activated currents, $I_{N a P}$ and $I_{h}$, which is described in Section 2.1.2 and Appendix B. Moreover, in this case we are able to provide an improvement of the linear estimation based on the quadratization explained in Section 2.2.3. Next, we develop both cases separately.

\subsection{Misestimations in the pyramidal cell model with an AHP and an LTS currents}

Let us consider the model described in Section 2.1.1. For the sake of comparison, we perform the estimation both under the presence of subthreshold-activated ionic channels (AHP and LTS) and without it. The case with both AHP and LTS off (already studied in Guillamon et al (2006)) is included for completeness and reference, but we are mainly interested in the experiment with either $I_{A H P}$ or $I_{L T S}$ on.

In Figure 1A, we show the total subthreshold current $\left(I_{A H P}+I_{L T S}\right)$ versus the rest of ionic currents when no applied current was added to the neuron model, that is $I_{a p p}=0$. Note that in the time interval $(62,100) \mathrm{ms}$ the value of the subthreshold currents dominates. More precisely, in the time interval $(77,100) \mathrm{ms}$, the sum of the rest of ionic currents almost vanishes and so the neuron has only subthreshold activity. These subthreshold-dominant time intervals can be better appreciated in Figure 1B, where the ad hoc index of dominance $\chi(t)$ (see equation (2)) is shown: index values below $-\sqrt{2} / 2$ indicate dominance of AHP currents whereas index values above $\sqrt{2} / 2$ indicate dominance of LTS currents. We recall that, for each time $t$, this index is a statistical measure calculated from the currents for all $I_{a p p}$. In Figure 1C subthreshold-dominant intervals are shaded over the membrane potential for $I_{a p p}=0$.

The representation of index $\chi$ in Figure 1B is useful to select different situations of activation of subthreshold currents, see also Figure 1C. In particular, we analyse (see Figure 2): a case where the AHP current prevails over the LTS current (we choose $t=85 \mathrm{~ms}$, see Figure 1B-C), and a case where the dominating current is LTS (we choose $t=95 \mathrm{~ms}$, see Figure 1B-C). Note that in both cases the traces of the currents lie clearly under the threshold. We have applied the linear estimation procedure explained in Section 2.2.1 both for $I_{A H P}$-dominance $(t=85 \mathrm{~ms})$ and $I_{L T S}$-dominance $(t=95 \mathrm{~ms})$.

When both $I_{A H P}$ and $I_{L T S}$ are inactivated, the estimation in the subthreshold regime is very accurate, as we predicted. Indeed, in Figure 2A, the actual and the estimated slopes (corresponding to the estimation of $1 / g_{\text {syn }}$ ) and intercepts (corresponding to the estimation of $V_{e f f}$ ) show a substantial agreement. 


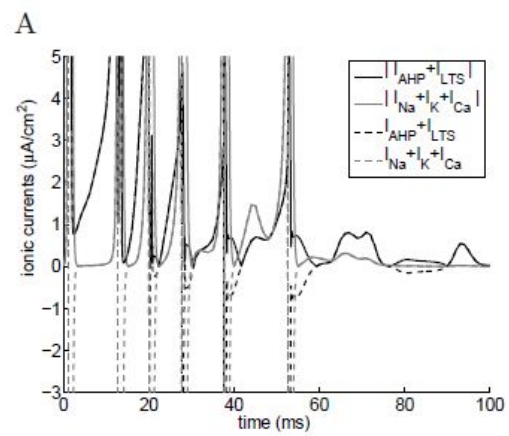

B

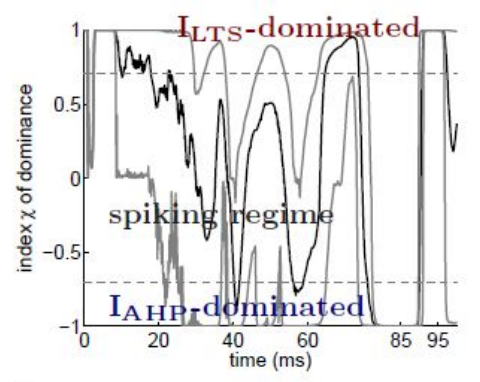

C

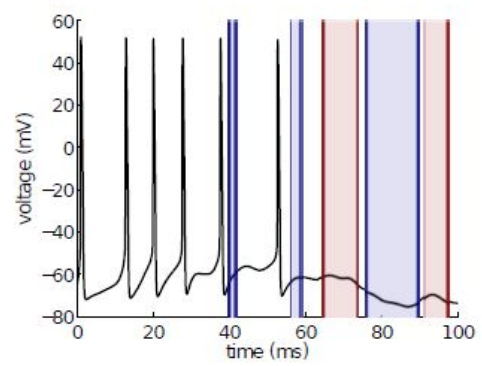

Figure 1: Representation of the subthreshold-activated ionic currents. In panel A, we compare the sum of the subthreshold currents, $-\left(I_{A H P}+I_{L T S}\right)$ (black trace), with the sum of all other ionic currents, $I_{C a}+I_{N a}+I_{K}$ (gray trace) with no applied current, that is $I_{a p p}=0$. The solid trace represents, in each case, the absolute value of the sums, whereas the dotted traces represent the actual values. We have chopped off the graphs for the sake of clarity. Panel B shows the mean (solid trace, in black) and the minimum and maximum values (solid traces, in gray) of the index of dominance of subthreshold currents over the different values of $I_{a p p}$. The two horizontal dotted lines (in gray) are the limits between the spiking and the nonspiking regimes and define three zones: a $I_{L T S}$-dominated non-spiking regime (upper zone), a spiking regime (middle zone) and an $I_{A H P}$-dominated non-spiking regime (lower zone). In panel $\mathrm{C}$, we show the $I_{A H P}$-dominated (bluish shadowed) and $I_{L T S^{-}}$ dominated (reddish shadowed) regimes obtained from panel B (mean across all applied currents) over the voltage course for $I_{a p p}=0$. 
The most interesting differences arise when we estimate the conductances under the presence of either $I_{A H P}$ or $I_{L T S}$, as shown in Figure 2B-C-D. Whereas both the slope $\left(1 / g_{s y n}\right)$ and the intercept $\left(V_{\text {eff }}\right)$ are well estimated with this kind of currents off (Figure 2A), the activation of any of them induces a mismatch between the theoretical $v-I_{a p p}$ line and the estimated one (see Figure 2B-C-D), where both the intercept and the slope are altered.

When the $I_{A H P}$ dominates (see Figure 2D), this situation clearly leads to an overestimation of the total synaptic conductance because the slope $\left(1 / g_{\text {syn }}\right)$ is underestimated; the effective reversal potential $\left(V_{e f f}\right)$ is also overestimated. On the other hand, when the dominating current is $I_{L T S}$ (see Figure 2B-C), the total synaptic conductance is underestimated whereas the effective reversal potential is overestimated. It is clear, then, that the solutions of the linear system (5) contain errors both in either $V_{\text {eff }}$ and $g_{s y n}$, thus indicating that the linear relationship hypothesis between $v_{f i l t}$ and $I_{a p p}$ can also be broken in (apparently) silent regimes. Interestingly, this effect was not detected when subthreshold currents are not considered (see Guillamon et al (2006)).

Using the estimations obtained from Figure 2 and applying the equations in (5), we can compute the relative errors in the estimation of synaptic conductances at critical time instants both when the dominating current is $I_{A H P}$ and $I_{L T S}$, see Table 1 .

\begin{tabular}{|c|c|c|c|c|}
\cline { 2 - 5 } \multicolumn{1}{c|}{} & \multicolumn{4}{c|}{ relative error } \\
\cline { 2 - 5 } \multicolumn{1}{c|}{} & $V_{\text {eff }}$ & $g_{\text {syn }}$ & $g_{E}$ & $g_{I}$ \\
\hline $\begin{array}{c}t=95 \mathrm{~ms} \\
\text { LTS } \\
\text { dominates }\end{array}$ & $3.42 \%$ & $-20.76 \%$ & $6.86 \%$ & $-29.32 \%$ \\
\hline $\begin{array}{c}t=95 \mathrm{~ms} \\
\text { LTS ON } \\
\text { AHP OFF }\end{array}$ & $6.17 \%$ & $-42.18 \%$ & $-16.59 \%$ & $-57.16 \%$ \\
\hline $\begin{array}{c}t=85 \mathrm{~ms} \\
\text { AHP } \\
\text { dominates }\end{array}$ & $0.74 \%$ & $11.85 \%$ & $44.51 \%$ & $13.29 \%$ \\
\hline
\end{tabular}

Table 1: Relative errors in the pyramidal cell model. For three different situations, $I_{L T S}$ dominance, $I_{A H P}$ dominance and only $I_{L T S}$ activated, we compute the relative error of the estimated effective reversal potential and the conductances with respect to the actual ones, that is $100\left(x_{\text {estimated }}-\right.$ $\left.x_{\text {actual }}\right) /\left|x_{\text {actual }}\right| \%$, where $x$ stands for $V_{\text {eff }}, g_{\text {syn }}, g_{E}$ and $g_{I}$. Rows 1,2 and 3 correspond, respectively, to panels $\mathrm{B}, \mathrm{C}$ and $\mathrm{D}$ of Figure 2. Moreover, row 1 corresponds to time $t=95 \mathrm{~ms}$ in the panels of Figure 3 and row 3 to time $t=85 \mathrm{~ms}$.

As explained above, in Figure 2 we have shown, for specific time values, how the activation of subthreshold ionic currents has an adverse effect on the estimations of synaptic conductances. However, these values constitute a too 
A

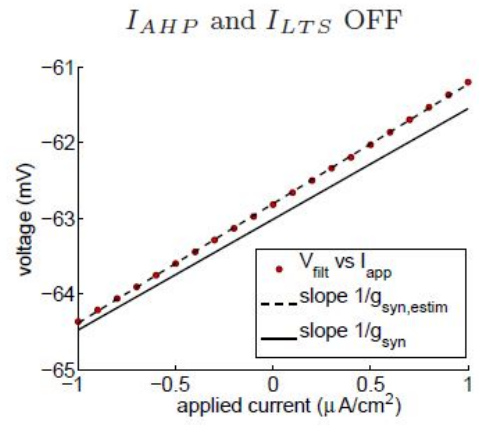

$\mathrm{C}$

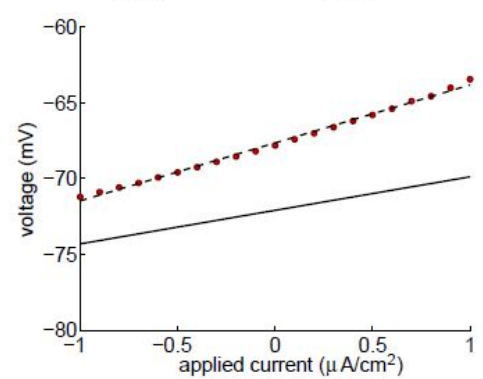

B

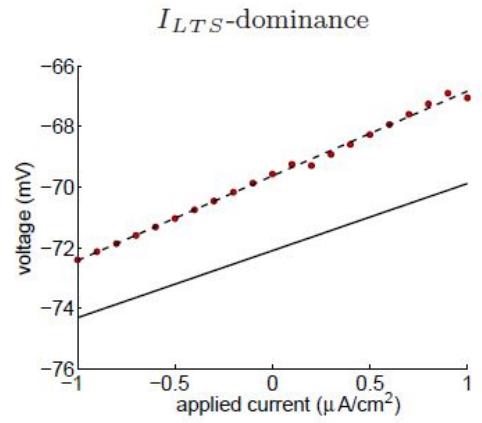

$\mathrm{D}$

$I_{A H P}$-dominance

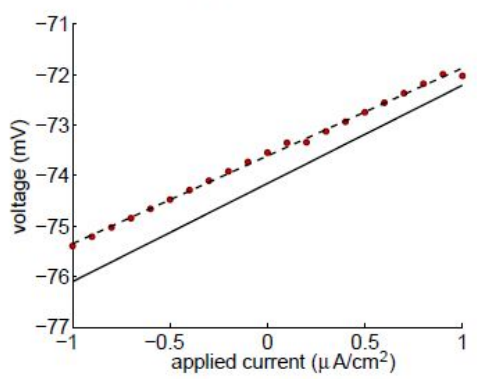

Figure 2: Comparison of the actual and estimated parameters for the pyramidal cell model under different dominance regimes. Panel A shows the estimation for time $t=70 \mathrm{~ms}$ (silent regime) when both subthreshold currents, $I_{A H P}$ and $I_{L T S}$, are inactive. Panels B and D show the estimation for times $t=95 \mathrm{~ms}$ ( $I_{L T S}$-dominated non-spiking regime) and $t=85 \mathrm{~ms}$ ( $I_{A H P}$-dominated non-spiking regime), respectively, when both $I_{A H P}$ and $I_{L T S}$ are active. Panel $\mathrm{C}$ shows a case where $I_{L T S}$ is activated but not the $I_{A H P}$. In all panels, the solid trace represents the theoretical regression line whereas the dotted trace is the straight line estimated from the data (red dots, which indicate the filtered membrane potential for different values of the applied current $\left.I_{a p p}\right)$. The relative errors in estimating $g_{s y n}, V_{e f f}, g_{E}$ and $g_{I}$ in panels $\mathrm{B}, \mathrm{C}$ and $\mathrm{D}$ are quantified in rows 1,2 and 3 of Table 1, respectively. 


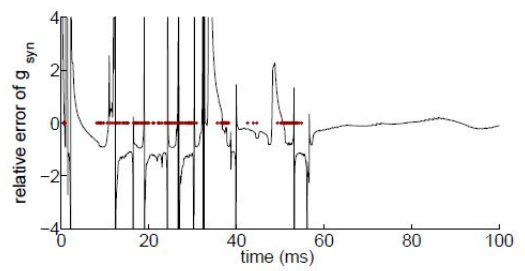

C

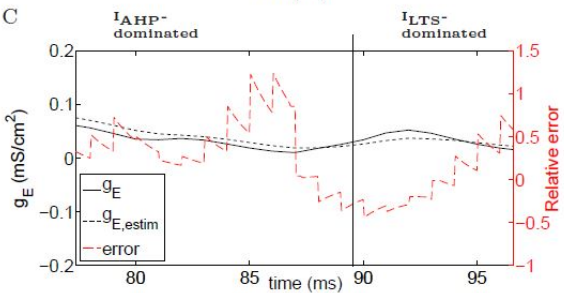

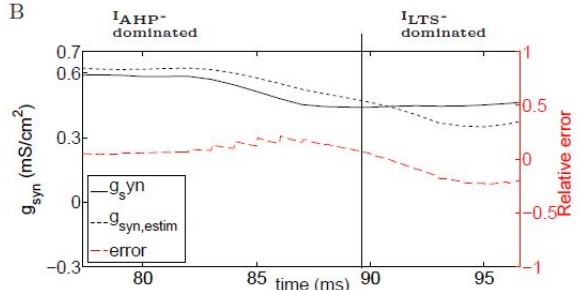

$\mathrm{D}$

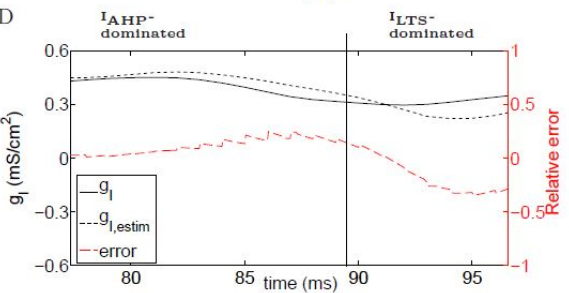

Figure 3: Relative errors in the $I_{A H P}$-dominated and $I_{L T S}$-dominated phases. Panel A shows the relative error of the synaptic conductance (solid trace) together with the spiking times (dots, in red) for each $I_{a p p}$ value in $\{-1,-0.9,-0.8, \ldots, 0.8,0.9,1\}$ in order to show the influence of spikes in the misestimations. Panels B, C and D show the relative error (dashed red trace), the estimated value (dotted black trace) and the actual value (solid black trace) of the total, excitatory and inhibitory synaptic conductances, respectively, in the subthreshold regime. Vertical lines show the border between the $I_{A H P^{-}}$and $I_{L T S}$-dominance phases, as in Figure 1C.

punctual examination of the problem; to show that these misestimations are maintained along a significative time interval, in Figure 3 we plot the actual conductances, the estimated ones and the relative errors for $t \in[77.35,96.6] \mathrm{ms}$ : panels $\mathrm{A}$ and $\mathrm{B}$ refer to $g_{\text {syn }}$ whereas panels $\mathrm{C}$ and $\mathrm{D}$ refer to $g_{E}$ and $g_{I}$, respectively. From panels B-C in Figure 1, we can see that this interval contains both an $I_{A H P}$-dominated subinterval (below $t=89.65 \mathrm{~ms}$ ) and a $I_{L T S}$-dominated subinterval (above $t=89.9 \mathrm{~ms}$ ). We discriminate the analysis according to these subintervals in order to discern the contamination due to the presence of the afterhyperpolarizing current from the presence of the low-threshold current. 


\begin{tabular}{|c|c|c|}
\cline { 2 - 3 } \multicolumn{1}{c|}{ MEAN } & $I_{A H P}$-dominated & $I_{L T S^{-}}$-dominated \\
\hline$g_{\text {syn }}$ & $8.6 \%$ & $-11.23 \%$ \\
\hline$g_{E}$ & $27.82 \%$ & $-2.85 \%$ \\
\hline$g_{I}$ & $10.12 \%$ & $-13.87 \%$ \\
\hline
\end{tabular}

\begin{tabular}{|c|c|c|}
\cline { 2 - 3 } \multicolumn{1}{c|}{ STD } & $I_{A H P}$-dominated & $I_{L T S}$-dominated \\
\hline$g_{s y n}$ & $6.45 \%$ & $9.69 \%$ \\
\hline$g_{E}$ & $35.14 \%$ & $30.48 \%$ \\
\hline$g_{I}$ & $9.07 \%$ & $16.30 \%$ \\
\hline
\end{tabular}

Table 2: Statistics of average relative errors for the pyramidal cell model. Statistics of relative errors in the estimation of total, excitatory and inhibitory synaptic conductances. The rows show the averages along time intervals and are computed $100\left(x_{\text {estimated }}-x_{\text {actual }}\right) /\left|x_{\text {actual }}\right| \%$, where $x$ stands for $g_{s y n}, g_{E}$ and $g_{I}$, respectively. Left column: averages over the time interval $[77.35,89.65] \mathrm{ms}$, where $I_{A H P}$ dominates; right column: averages over the time interval $[89.65,96.6] \mathrm{ms}$, where $I_{L T S}$ dominates, see also Figure 3 for reference.

\subsubsection{Estimation errors in the $I_{A H P}$-dominated time interval}

The explanation for the influence of this kind of currents in the estimations can be found in the time scale of activation, namely the long-time scale of $\left[\mathrm{Ca}^{2+}\right]$. It turns out that the $I_{A H P}$ has a strong influence during around $55 \mathrm{~ms}$ after the spike (since $\tau_{C a}=80 \mathrm{~ms}$ and $\left[\mathrm{Ca}^{2+}\right]$ has an exponential decay, see equation (13) in Appendix A). Then, it may happen that, for some $I_{a p p}$, the $I_{A H P}$ current is still influencing while for other $I_{a p p}$, the $I_{A H P}$ is negligible at this moment in time. This fact leads to a breaking of the linearity of the $I_{a p p}-v_{f i l t}$ relationship. Observe (see Figure 1A) that spiking regimes finish around $t=53 \mathrm{~ms}$ so that the $I_{A H P}$-dominance interval, $t \in[77.35,89.65] \mathrm{ms}$, is coherent with the time scale of this calcium-induced potassium channel.

For this time interval, we observe (see Table 2) an average error in the total synaptic conductance around $8.64 \pm 6.45 \%$, that is somehow preserved for the inhibitory conductance $(10.12 \pm 9.07 \%)$ but a notable increase of the excitatory conductance error up to $27.82 \pm 35.14 \%$. Our results confirm and quantify the discrepancies between the actual and estimated histograms of $g_{A M P A}$ and $g_{G A B A}$ observed in Figure 6 of Rudolph et al (2004) under the presence of subthreshold-activated ionic channels. Our quantitative analysis shows that these errors cannot be disregarded and that they can lead to wrong conclusions about the reconstruction of $g_{E}$ and $g_{I}$ temporal profiles.

\subsubsection{Estimation errors in the $I_{L T S}$-dominated time interval}

The $I_{A H P}$ current needs spiking activity before being activated and it could be argued that the observed errors are due to this post-influence of the spiking misestimations rather than the presence of subthreshold ionic channels. To 
A

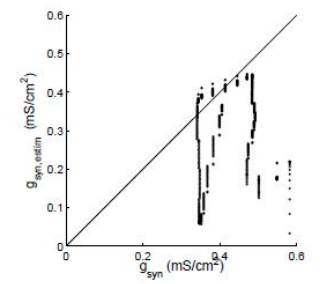

B

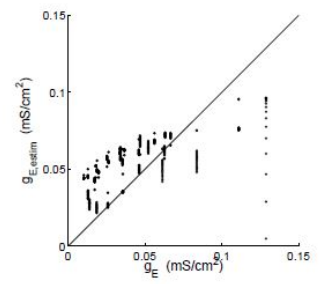

C

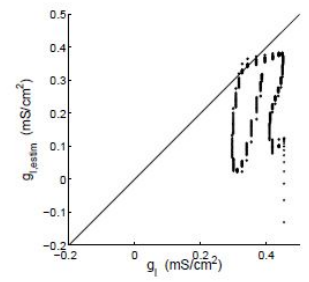

Figure 4: Actual versus estimated conductances for the pyramidal cell model with only $I_{L T S}$ active. Panels A, B and C represent the scatter plot of the actual versus the estimated total, excitatory and inhibitory synaptic conductances, respectively, for a time interval where only the $I_{L T S}$ current is active. The identity line has been added on the scatter plot as a reference to compare how the estimated conductances agree with the actual ones.

ensure that this is a pure subthreshold effect, we have chosen another type of subthreshold-activated channel, a low-threshold one which activates in a range of voltage values still far from the spiking threshold but sufficiently above the hyperpolarized state.

For this time interval, we observe an average error in the total synaptic conductance around $-11.23 \pm 9.69 \%$, that become somehow steady for the inhibitory conductance $(-13.87 \pm 16.30 \%)$ and a low mean disperse estimation for the excitatory conductance $(-2.85 \pm 30.48 \%)$.

Considering the $I_{A H P}$ current inactive, Figure 4 shows the actual and the estimated conductances when only the $I_{L T S}$ current is active, that is $t \in[70,100]$. In this plot we can appreciate the misestimations of the linear regression even in the subthreshold regime since, the dots of the scatter plot do not tend to align along the identity line.

\subsubsection{Source of misestimations}

We know by previous studies, see Guillamon et al (2006), that the mismatches of the estimation in the spiking regime come from the wrong assumption that the different ionic currents vanish. When either AHP or LTS currents are on, the errors in the estimation spread to the regimes where these currents are active since we can not suppose that they vanish. Therefore the linear regression (4) should be corrected as

$$
v\left(t ; I_{a p p}\right)=V_{e f f}(t)-\frac{I_{A H P}(t)+I_{L T S}(t)}{g_{s y n}(t)}+\frac{I_{a p p}}{g_{s y n}(t)} .
$$

If we examine the effect of the new term $I_{A H P}(t)+I_{L T S}(t)$ in equation (10) on the solution of the linear regression (5), we obtain that the solution is modified by adding an extra term depending on the subthreshold currents to 
the $g_{E}$ and $g_{I}$ expressions:

$$
\begin{aligned}
& g_{E}=\frac{\left(g_{s y n}-g_{L}\right) V_{I}-V_{e f f} g_{s y n}+g_{L} V_{L}}{V_{I}-V_{E}}-\frac{I_{A H P}+I_{L T S}}{V_{I}-V_{E}} \\
& g_{I}=\frac{-\left(g_{s y n}-g_{L}\right) V_{E}+V_{e f f} g_{s y n}-g_{L} V_{L}}{V_{I}-V_{E}}+\frac{I_{A H P}+I_{L T S}}{V_{I}-V_{E}}
\end{aligned}
$$

The cause of the pitfalls of the estimation are thus due to ignore the $I_{A H P}(t)+$ $I_{L T S}(t)$ term in the linear estimation procedure. Basically three different situations may arise: (a) if $I_{A H P}+I_{L T S}$ were constant with respect to $I_{a p p}$, then we would obtain a perfect fit and estimation of $g_{s y n}$, whereas $V_{\text {eff }}$ would be misestimated; (b) if $I_{A H P}+I_{L T S}$ were to vary linearly with respect to $I_{a p p}$, then the fit would remain perfect, but both $g_{s y n}$ and $V_{\text {eff }}$ would be misestimated; (c) otherwise, neither the fit would be good and the estimations trustable.

In the first two cases ((a) and (b)) one could devise a way to foresee whether $V_{\text {eff }}$ and $g_{\text {syn }}$ are underestimated or overestimated. For instance, in the AHPdominated regime, since $I_{A H P}+I_{L T S}<0$ (see Figure 1B), the $\left(I_{a p p}, v\left(t ; I_{a p p}\right)\right)$ points obtained from the experiments would be distributed above the line corresponding to the ideal situation where no ionic currents are active. For case (a), this would lead to an overestimation of $V_{\text {eff }}$ and, for case (b), to an overestimation of $V_{\text {eff }}$ and an underestimation (resp., overestimation) of $g_{s y n}$ if the $\left(I_{A H P}+I_{L T S}\right)$ versus $I_{a p p}$ slope is positive (resp., negative). Unfortunately, the most common case is (c), in which the above predictions can be taken only as an orientation. Indeed, in Table 1 we can observe, for instance, underestimations of $V_{\text {eff }}$ in the $A H P$-dominate regime. The same analysis can be applied to the estimations of $g_{E}$ and $g_{I}$ from $g_{s y n}$ and $V_{\text {eff }}$ (see equation (11)), so we cannot assess a general relationship between $A H P$ or LTS domination and the sign of the misestimations.

\subsubsection{Linearization of the subthreshold ionic currents approach}

In the case of $L T S$, this current is activated on the subthreshold regime independently of the spikes. Its characteristic time scale $\tau_{L T S}$ changes according to the steady state value of the current which is given by (12) with $w=h_{L T S}$.

As we have mentioned in Section 2.2.2, during the phase where $I_{L T S}$ is dominating, the plot of the function

$$
I_{L T S, \infty}(v):=g_{L T S} m_{L T S, \infty}(v)^{3} h_{L T S, \infty}(v)\left(v-V_{C a}\right)
$$

is not far from linear. Then, $I_{L T S, \infty}(v)$ can be linearised for $v \approx[-75,-60] m V$ and the estimation described in Section 2.2.2 can be done. However, this approach did not lead to good estimations either, the reason being the slow approach of the $h_{L T S}$ variable to its steady state $h_{L T S, \infty}(v)$ (see Figure 5A). Anyway, we found interesting to show the slight improvement obtained following this approach. Just as a proof of concept, we tried with a 10-times smaller time constant, namely $\tau_{L T S}=4.5 \mathrm{~ms}$, in which case $h_{L T S}$ catches $h_{L T S, \infty}(v)$ up sufficiently fast to be close enough during the $I_{L T S}$-dominance phase (see Figure 5B). Thus we get that $h_{L T S}$ closer to $h_{L T S, \infty}(v)$ in the interval in which 
A

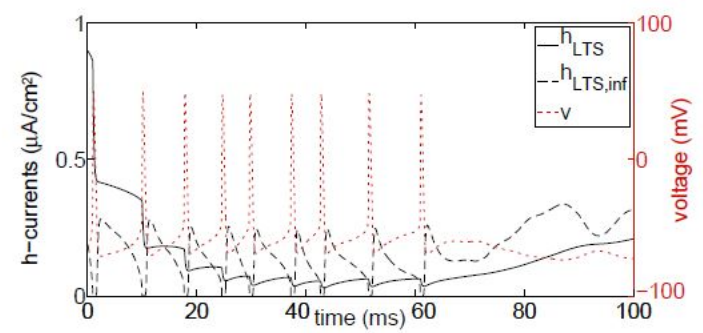

B

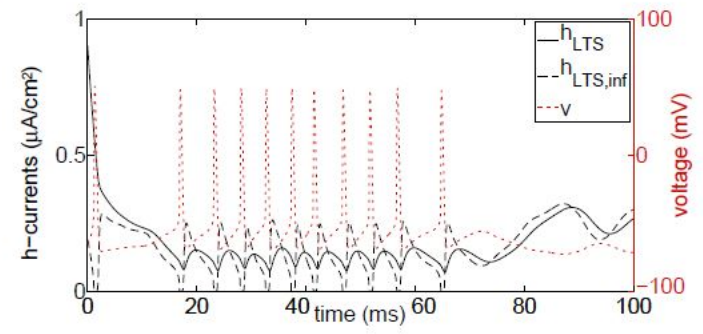

Figure 5: Behaviour of $h_{L T S}$ and $h_{L T S, \infty}(v)$ Both panels show the voltage of the neuron (dotted red trace) and the behaviour of $h_{L T S}$ (solid black trace) and of $h_{L T S, \infty}(v)$ (dashed black trace) over time. In panel $\mathrm{A}$ we can see how $h_{L T S}$ and $h_{L T S, \infty}(v)$ do not match when using the characteristic time scale of the system. Otherwise, in panel B, where the parameter $\tau_{L T S}$ has been fixed to be 4.5 , we observe that $h_{L T S}$ reaches $h_{L T S, \infty}(v)$ very quickly. 
$I_{L T S, \infty}(v)$ is linear and so $I_{L T S, \infty}(v) \approx I_{L T S}(m, h, v)$. Then, the procedure proposed at the end of Section 2.2.2 can be applied to improve the linear estimation of the synaptic conductances. Obviously we are certainly loosing biophysical interest since this would be a valid approach only for putative "fast low-threshold" channels, which are seldomly reported in the literature, see Carbone et al (2006). In this case, as we can see in Figure 6, the agreement of the estimated data with the actual data presents an important improvement.

\subsection{Misestimations in the stellate cell model with NaP- and h- currents}

In the previous section we have seen how the subthreshold-activated currents lead the linear estimation to significant errors. Let us now consider the stellate cell model described in Section 2.1.2 to stress the misestimations of the linear regression under the presence of currents, which cause oscillatory activity in the subthreshold regime and nonlinear effects. Finally, we are presenting an improvement of the linear estimation to take into account this nonlinearities and the fact that the ionic currents may not reach the steady state, where the alternative presented in Section 3.1.4 fails.

In contrast to the pyramidal cell model, the model we are currently considering has no presence of currents that lead the neuron to fire. For this reason, the goodness of the estimation can not be affected by the presence of spiking currents, but only for the subthreshold ones. In order to proceed with the linear estimation, we check that, for values of $I_{a p p} \in[-4,-3]$, both subthreshold currents are active. Their magnitudes oscillate between 1 and $7 \mu \mathrm{A} / \mathrm{cm}^{2}$ for the $I_{N a P}$, and with magnitude between 4 and $7 \mu \mathrm{A} / \mathrm{cm}^{2}$ for the $I_{h}$ (see Figure 7 for a representation of $I_{N a P}$ and $I_{h}$ when $\left.I_{a p p}=-3.5\right)$.

Figure 8 shows the results of applying the standard linear estimation procedure defined by formulas (4) and (5). Upper panels show how the estimated synaptic conductances (either the total one or both excitatory and inhibitory) clearly diverge from the actual ones along time. Lower panels of Figure 8 contain the scatter plots of the set of paired points $\left(g_{\text {actual }}, g_{\text {estimated }}\right)$ for the total, excitatory and inhibitory conductances. The arrangement of the points far from the identity line gives a clear evidence of the non-validity of the standard linear estimation procedure.

Finally, in Figure 9, we show the membrane potential computed by using the actual conductances together with the membrane potential obtained with the estimated conductances (the reconstructed voltage). Comparing the results obtained in this figure and the time course of the ionic currents (see Figure 7), we can see how the reconstructed voltage is worse when subthreshold currents present higher activity, as it is also noticeable for the synaptic conductances.

Summing up, te results shown for the stellate cell model up to this point, together with those obtained for the pyramidal cell model with $I_{A H P}$ and $I_{L T S}$ currents, clearly demonstrate that the standard linear estimation procedure turns out to be inappropriate as well in subthreshold regimes. For the pyramidal cell model, we have come up with a modified linear regression which mildly 
A

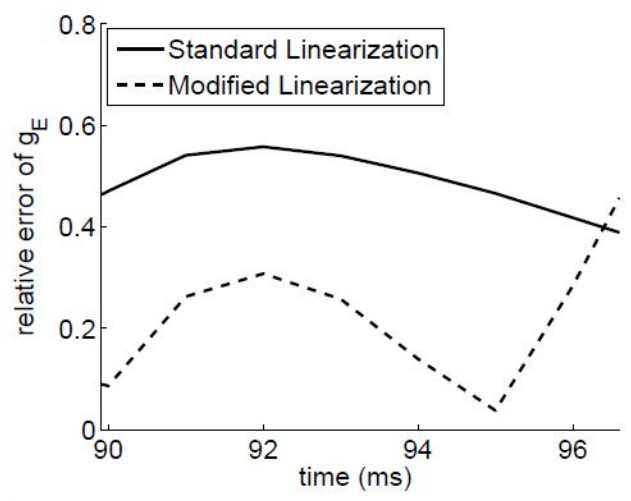

$\mathrm{B}$

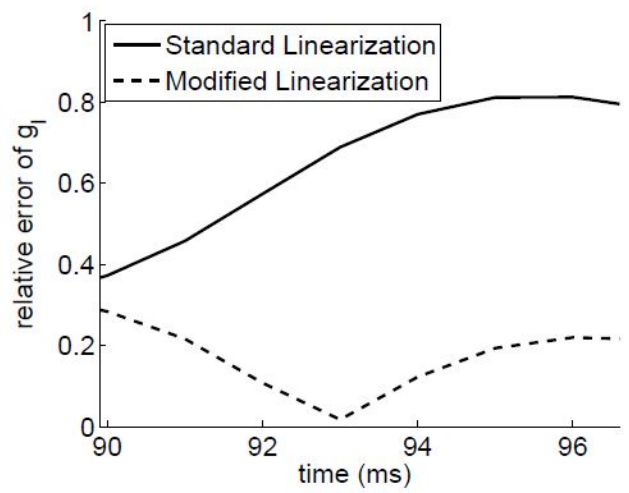

Figure 6: Estimation of excitatory and inhibitory conductances using a rectified linear regression. For fast low-threshold dynamics $\left(\tau_{L T S}=4.5 \mathrm{~ms}\right)$, panels $\mathrm{A}$ and B show the relative errors of $g_{E}$ and $g_{I}$, respectively, when the standard linear estimation (using (4) and (5)) has been used (solid trace) and when the estimation has been modified using the procedure proposed at the end of Section 2.2.2 (dashed trace). 


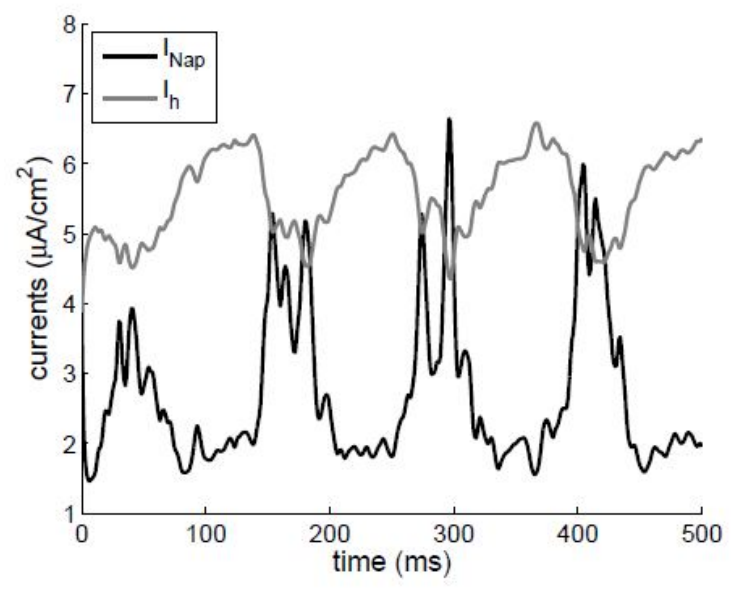

Figure 7: Representation of the subthreshold-activated ionic currents in the stellate cell model. Activity of the two active subthreshold currents in this model, the persistent sodium current (black trace) and the h-current (gray trace) for a central value of the set of applied currents used in the estimation, that is, $I_{a p p}=-3.5$.

improved the estimations. For the stellate cell model, taking advantage of the minimal model reduction given in Rotstein (2015), we are able to propose a promising nonlinear estimation procedure, see Section 2.2.3, that improves the estimations by more than one order of magnitude.

\subsubsection{Quadratization alternative}

Following the procedure presented in Section 2.2.3, from the total synaptic current, we can discern between excitatory and inhibitory conductances using two trials corresponding to different applied currents. In this section we want to show the goodness of this new approach.

Having two different voltage traces for different applied currents, from equation (8) one can estimate the total synaptic current for each trial. In Figure $10 \mathrm{~A}$, we can appreciate how the estimated synaptic current fits to the actual one along time. The scatter plot presented in panel B illustrates how the estimated and the actual values are concentrated in the vicinity of the identity line, which means a good estimation of the synaptic current.

Using two different applied currents, say $I_{a p p, 1}$ and $I_{a p p, 2}$, and the corresponding voltage traces $v_{1}(t)$ and $v_{2}(t)$, we obtain $I_{s y n, 1}(t)$ and $I_{s y n, 2}(t)$, respectively, from equation (8), see also Figure 10. Then, using (9), we obtain an estimation of the time course of the excitatory and the inhibitory conductances which are shown and compared with the actual ones in Figure 11. In the upper panels of this figure, one can see how the actual and the estimated traces fit better than in the linear regression case. Moreover, the estimation is better 
A

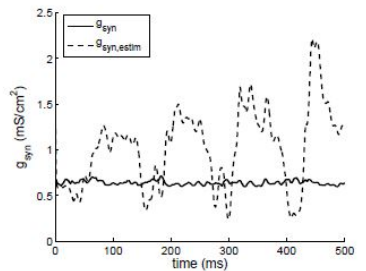

D

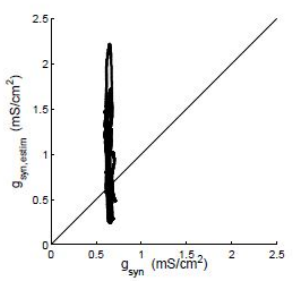

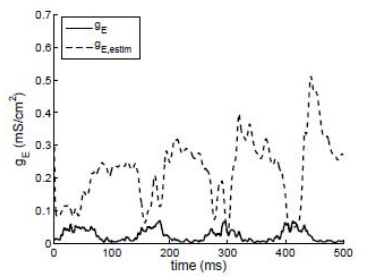

$\mathrm{E}$

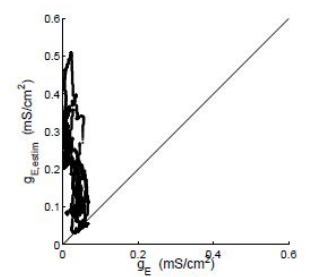

$\mathrm{C}$

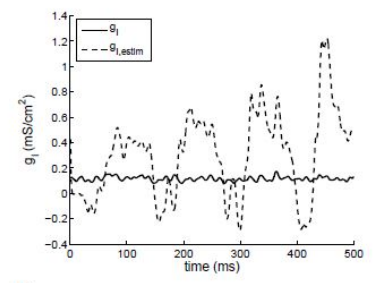

$\mathrm{F}$

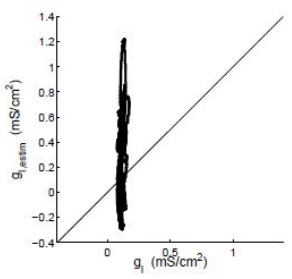

Figure 8: Comparison of the actual and the linearly estimated conductances for the stellate cell model. Upper panels show the actual time course (solid black traces) and the estimated time courses (dotted black traces) of the total (panel A), the excitatory (panel B), and the inhibitory (panel C) synaptic conductances when the linear estimation has been applied for 21 values of $I_{a p p} \in[-4,-3]$, equispaced. Low panels represent the scatter plot of the actual conductances versus the estimated. The plotted conductances are the synaptic one, the excitatory one, and the inhibitory one (from left to right). The identity line has been added on the scatter plot to compare how the estimated conductances disagree with the actual ones. 


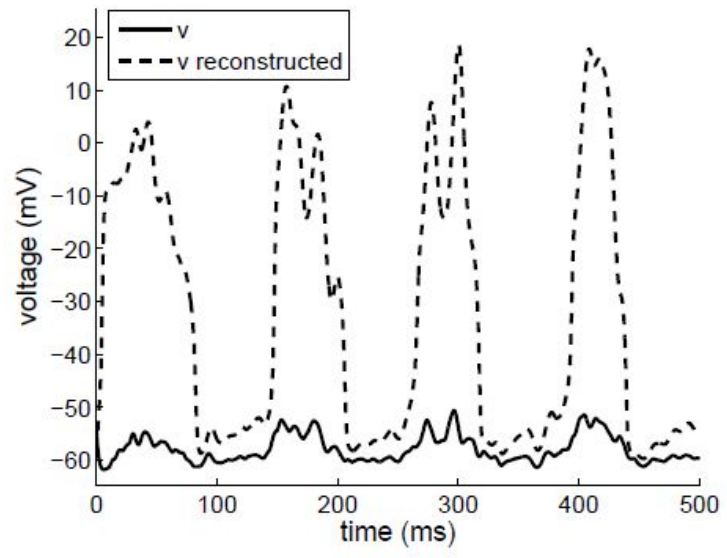

Figure 9: Voltage dynamics generated by both the actual and the linearly estimated conductances in the stellate cell model. Solid black trace represents the voltage obtained with the actual conductances while the dotted black trace represents the reconstructed voltage, obtained by plugging the estimated conductances into the model. The applied current considered in both cases is $I_{a p p}=-3.5$.

for the excitatory conductances than for the inhibitory ones, as it can also be seen in the lower panels, where the scatter plot presents higher concentration on the vicinity of the identity line for the $g_{E}$ case. In Table 3 , we give a complete quantitative description of the errors of the estimation both for the linearization and the quadratization procedures.

\begin{tabular}{|c|c|c|}
\cline { 2 - 3 } \multicolumn{1}{c|}{ MEAN } & Linearization & Quadratization \\
\hline$g_{\text {syn }}$ & $61.73 \%$ & $-0.46 \%$ \\
\hline$g_{E}$ & $2335.03 \%$ & $-4.92 \%$ \\
\hline$g_{I}$ & $155.68 \%$ & $-1.06 \%$ \\
\hline
\end{tabular}

\begin{tabular}{|c|c|c|}
\cline { 2 - 3 } \multicolumn{1}{c|}{ STD } & Linearization & Quadratization \\
\hline$g_{\text {syn }}$ & $69.79 \%$ & $4.35 \%$ \\
\hline$g_{E}$ & $2507.84 \%$ & $30.84 \%$ \\
\hline$g_{I}$ & $287.09 \%$ & $20.94 \%$ \\
\hline
\end{tabular}

Table 3: Statistics of average relative errors for the stellate cell model. Statistics of relative errors in the estimation of total, excitatory and inhibitory synaptic conductances. The rows show the averages along time intervals and are computed $100\left(x_{\text {estimated }}-x_{\text {actual }}\right) /\left|x_{\text {actual }}\right| \%$, where $x$ stands for $g_{\text {syn }}, g_{E}$ and $g_{I}$, respectively. Left column: averages corresponding to the Linearization procedure; right column: averages corresponding to the Quadratization procedure. 
A

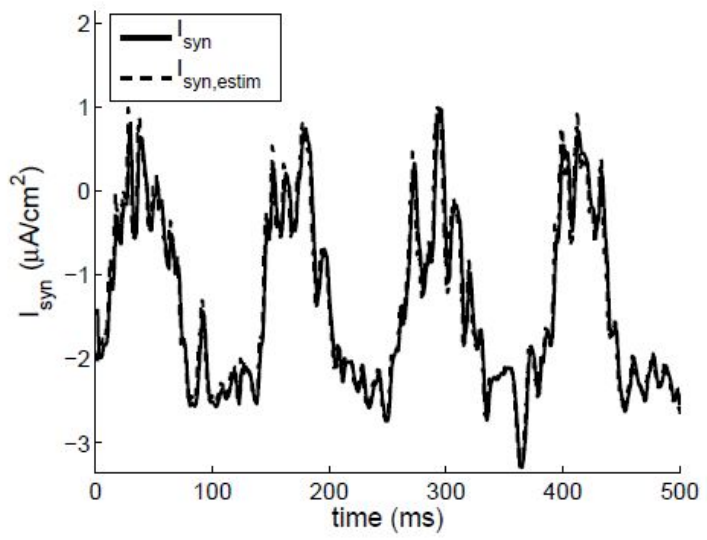

B

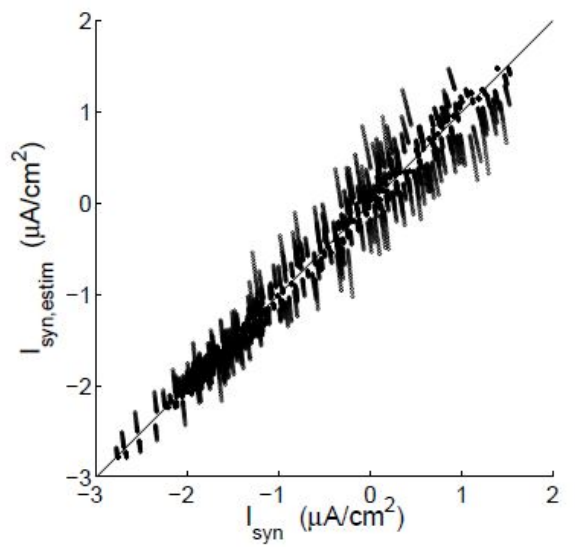

Figure 10: Actual synaptic currents compared to those estimated through the quadratizaton procedure. Panel A shows the time course of the actual synaptic current (solid black traces) and the estimated one (dotted black traces) when the quadratization approach has been applied for $I_{a p p}=-3.5$. Panel B represents the scatter plot of the actual synaptic current versus the estimated. The identity line has been added on the scatter plot to compare how the estimated values agree with the actual ones. 
A

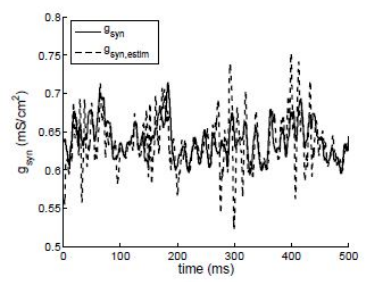

D

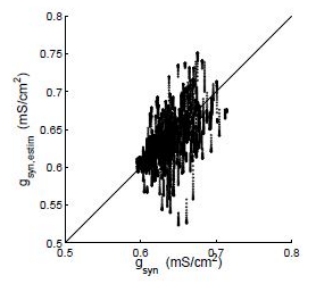

B
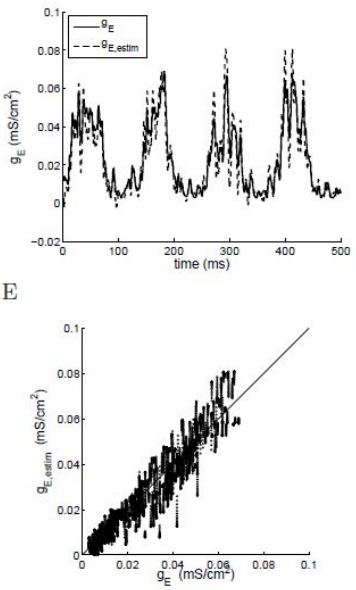

C

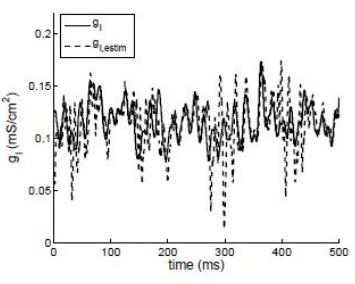

$\mathrm{F}$

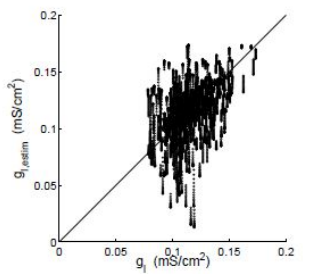

Figure 11: Comparison of the actual and estimated conductances using the quadratization approach. Upper panels show the actual time course (solid black traces) and the estimated time courses (dotted black traces) of the total (panel A), the excitatory (panel B), and the inhibitory (panel C) synaptic conductances when the quadratization approach is applied for $I_{a p p}=-4$ and $I_{a p p}=-3.5$. Low panels represent the scatter plot of the actual conductances versus the estimated ones, showing a good agreement.

Finally, to study the effect of the errors done in the estimation, we reconstruct the voltage traces of the neuronal model using the estimated conductances. As we can see in Figure 12, both the actual and the reconstructed voltages do not present big changes, contrary to the case when linear regression is applied.

These results imply that the quadratization approach is a better alternative to estimate conductances when nonlinear activity coming from subthresholdactivated ionic currents is present.

\section{Discussion}

It is well-known that linear estimations of synaptic conductances are not trustable when data is extracted intracellularly from spiking activity of neurons, see Guillamon et al (2006). Data from experimental studies in the current literature are generally treated taking this cautious message into account but the probable presence of subthreshold-activated currents confounding the impact of presynaptic activity is yet generally neglected. Our study entails that estimation of synaptic conductances in the presence of subthreshold-activated currents, even obtained from periods of apparently silent activity, may not be accurate. We have explored this feature using first a computational model with either 


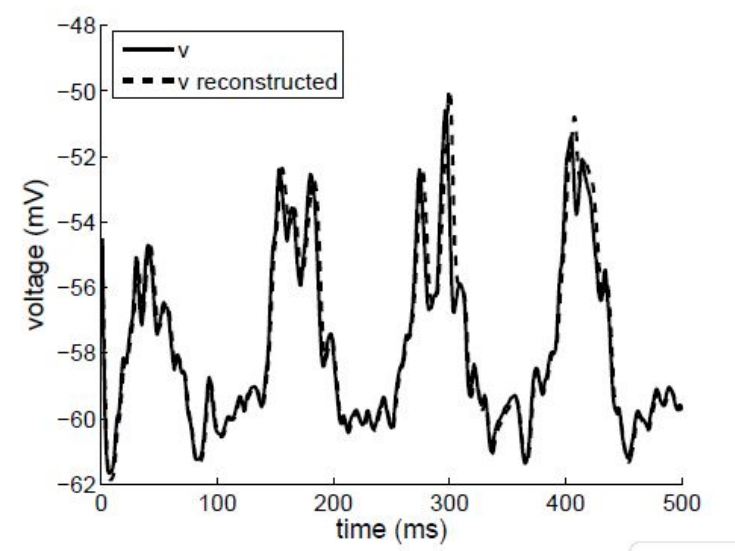

Figure 12: Voltage dynamics generated by both the actual and the quadratically estimated conductances in the stellate cell model. Solid black trace represents the voltage obtained with the actual conductances while the dotted black trace represents the reconstructed voltage, obtained by plugging the estimated conductances into the model. The applied current considered in both cases is $I_{a p p}=-3.5$.

afterhyperpolarizing or low-threshold activated currents and both sources of subthreshold activity lead to similar conclusions. To strengthen the message, a second model has been taken into account, consisting of two different activatedsubthreshold currents, a resonant one $\left(I_{h}\right)$ and a persistent one $\left(I_{\mathrm{NaP}}\right)$. In the first computational model, the pyramidal model described in Section 2.1.1, we have been able to isolate periods of activity where either AHP or LTS currents dominate. In the first case, the long-time scale of $\left[\mathrm{Ca}^{2+}\right]$ provides an AHP-dominated time window (55 $\mathrm{ms}$ approximately) after the spiking activity in which linear estimations of synaptic conductances fail. One might argue that, since AHP is only present after spiking activity, this failure is a natural extension of bad estimations in spiking regimes rather than an effect of subthreshold activity. To rule out this interpretation, we have been considered both examining the periods where LTS currents dominate, which have no dependence on previous spiking activity, and the second model, the stellate model described in Section 2.1.2, with no mechanisms for spiking. In both cases we observed similar misestimations, being the main explanation is the loss of linearity in the $I-V$ relationship. Thus, we conclude that it is an ubiquitous feature in subthresholdactivated currents. Therefore, our findings add a new warning message for the treatment of data obtained from intracellular recordings and the conclusions that can be drawn from them, specially those concerning the balance between excitation and inhibition.

In this paper we have also considered possible improvements to get more accurate estimations of the synaptic conductances. In particular, we also provide a strategy based on a quadratization of the neuronal model presented in Rotstein 
(2015). We think that this finding suggests experimental outcomes. Apart from the obvious alternative of a pharmacological block of all possible subthresholdactivated channels, which indeed could lead to a too passive integrator, we propose a fitting of the data to a quadratic model that could account for channels whose underlying dynamics is similar to that of the stellate cell model presented in this paper.

This quadratization approach still allows for further improvements that we plan to tackle. The main point is that the method lowers its performance when applied to synaptic inputs with faster time-scales. In our examples, we have used as synaptic drive the input of a realistic network on a single cell, thus including different synaptic receptors types and so a wide range of synaptic time-scales (in Figure 11-ABC it can be noticed that the synaptic input contains rapid fluctuations). Therefore, although the overall estimation is already excellent, probably due to an important role of slow synaptic components, it would be possible to improve the estimation of these fast components. We think that a deeper study using singular perturbation theory would help in getting a more refined version of the method.

One could also add other misestimation sources, specially those emanated from the dendro-somatic interaction (see for instance Cox (2004)), but our goal in this work is to observe the net effect of the presence of subthreshold currents; accordingly, we have considered single-compartment neuron models, thus assuming that the voltage does not vary from the dendrite, where the signal is received by the neuron, to the soma, where we are focusing on.

Unfortunately, the use of linear estimation methods to extract synaptic conductance time courses has been profusely used in experimental studies (for brief illustrations see Anderson et al (2000), Wehr and Zador (2003) and Bennett et al (2013)) and important conclusions about brain's functionality have been drawn from the excitatory-inhibitory separation of these time courses. Our results imply, at least, that caution has to be applied in trusting this type of results and, probably, a revision of functionality conclusions obtained from experimental data should be conducted.

Besides the implications on experimental data treatment, our findings also bring up new arguments to extend previous theoretical approaches, see for instance Rudolph et al (2004), that rely on the formulation of the dynamics of the neuron as an integrate \& fire model. More specifically, new analytical methods to obtain Fokker-Planck-like equations for integrate \& fire models with subthreshold-activated currents should be devised. This is a rather challenging problem both from the point of view of stochastic differential equations and numerical integration of partial differential equations.

To sum up, we would like to emphasize our message that, in the estimation of conductance in subthreshold regimes, one should also rule out the presence of ionic currents before proceeding with linear estimations methods. Otherwise, as an alternative method to estimate the conductances, the quadratization approach described in Section 2.2.3 should be used in order to minimize the errors. 


\section{Acknowledgements}

AG is supported by the MINECO grant MTM2012-31714 (DACOBIANO) and the Generalitat de Catalunya grant AGAUR 2014SGR-504. CV is supported by the MCYT/FEDER grant MTM2011-22751 and MICINN/FEDER grant MTM2014-54275-P. We are grateful to Louis Tao for providing us the dataset of testing conductances and also to the high-performance parallel computing cluster Eixam, at the Dept. of Applied Mathematics I (UPC), www.ma1.upc.edu/ eixam/index.html.

\section{References}

Anderson JS, Carandini M, Ferster D (2000) Orientation tuning of input conductance, excitation, and inhibition in cat primary visual cortex. Journal of neurophysiology 84(2):909-926, URL http://jn.physiology.org/content/84/2/909.abstract.

Bédard C, Béhuret S, Deleuze C, Bal T, Destexhe A (2011) Oversampling method to extract excitatory and inhibitory conductances from single-trial membrane potential recordings. Journal of neuroscience methods DOI 10.1016/j.jneumeth.2011.09.010.

Bennett C, Arroyo S, Hestrin S (2013) Subthreshold mechanisms underlying state-dependent modulation of visual responses. Neuron 80(2):350 - 357, DOI http://dx.doi.org/10.1016/j.neuron.2013.08.007.

Berg RW, Ditlevsen S (2013) Synaptic inhibition and excitation estimated via the time constant of membrane potential fluctuations. Journal of Neurophysiology 110(4):1021-1034, DOI 10.1152/jn.00006.2013.

Carbone E, Marcantoni A, Giancippoli A, Guido D, Carabelli V (2006) Ttype channels-secretion coupling: evidence for a fast low-threshold exocytosis. Pflügers Archiv 453(3):373-383, DOI 10.1007/s00424-006-0100-7.

Closas P (2014) Sequential estimation of neural models by bayesian filtering. diploma thesis, FME-Univ. Politènica de Catalunya

Cox SJ (2004) Estimating the location and time course of synaptic input from multi-site potential recordings. J of Computational Neuroscience 17:225-243.

Destexhe A, Babloyantz A, Sejnowski T (1993) Ionic mechanisms for intrinsic slow oscillations in thalamic relay neurons. Biophysical Journal 65:1538-1552.

Destexhe A, Rudolph M, Paré D (2003) The high-conductance state of neocortical neurons in vivo. Nature Reviews Neuroscience 4(9):739-751, DOI 10.1038/nrn1198. 
Dickson CT, Magistretti J, Shalinsky MH, Fransén E, Hasselmo ME, Alonso A (2000) Properties and role of $\mathrm{I}(\mathrm{h})$ in the pacing of subthreshold oscillations in entorhinal cortex layer II neurons. Journal of neurophysiology 83(5):25622579, URL http://view.ncbi.nlm.nih.gov/pubmed/10805658.

Fenichel N (1979) Geometric singular perturbation theory for ordinary differential equations. Journal of Differential Equations 31(1):53-98.

Guillamon A, McLaughlin DW, Rinzel J (2006) Estimation of synaptic conductances. Journal of Physiology-Paris 100(1-3):31-42, DOI 10.1016/j.jphysparis.2006.09.010.

Hirsch JA, Alonso JM, Reid CR, Martinez LM (1998) Synaptic Integration in Striate Cortical Simple Cells. Journal of Neuroscience 18(22):9517-9528, URL http://www.jneurosci.org/cgi/content/ abstract/ 18/22/9517.

Hotson JR, Prince DA (1980) A calcium-activated hyperpolarization follows repetitive firing in hippocampal neurons. J Neurophysiology 43(2):409-419, URL http://jn.physiology.org/content/43/2/409.full.

Kobayashi R, Tsubo Y, Lansky P, Shinomoto S (2011) Estimating time-varying input signals and ion channel states from a single voltage trace of a neuron. Advances in Neural Information Processing Systems (NIPS) 24:217-225.

Lankarany M, Zhu WP, Swamy M, Toyoizumi T (2013a) Blind deconvolution of hodgkin-huxley neuronal model. In: Engineering in Medicine and Biology Society (EMBC), 2013 35th Annual International Conference of the IEEE, pp 3941-3944, DOI 10.1109/EMBC.2013.6610407.

Lankarany M, Zhu WP, Swamy MNS, Toyoizumi T (2013b) Inferring trial-totrial excitatory and inhibitory synaptic inputs from membrane potential using Gaussian mixture Kalman filtering. Frontiers in Computational Neuroscience 7, DOI 10.3389/fncom.2013.00109.

Lombardi F, Herrmann HJ, Perrone-Capano C, Plenz D, de Arcangelis L (2012) Balance between excitation and inhibition controls the temporal organization of neuronal avalanches. Phys Rev Lett 108:228,703, DOI 10.1103/PhysRevLett.108.228703.

McLaughlin D, Shapley R, Shelley M, Wielaard DJ (2000) A neuronal network model of macaque primary visual cortex (V1): orientation selectivity and dynamics in the input layer 4Calpha. Proceedings of the National Academy of Sciences of the United States of America 97(14):8087-8092, DOI $10.1073 /$ pnas. 110135097 .

Monier C, Fournier J, Fregnac Y (2008) In vitro and in vivo measures of evoked excitatory and inhibitory conductance dynamics in sensory cortices. Journal of Neuroscience Methods 169(2):323-365, DOI 10.1016/j.jneumeth.2007.11.008. 
Paninski L, Vidne M, DePasquale B, Ferreira DG (2012) Inferring synaptic inputs given a noisy voltage trace via sequential monte carlo methods. Journal of Computational Neuroscience 33(1):1-19, URL http://link.springer.com/article/10.1007/ s10827-011-0371-7.

Pospischil M, Piwkowska Z, Bal T, Destexhe A (2009) Extracting synaptic conductances from single membrane potential traces. Neuroscience (158):545552 .

Rotstein H (2015) Subthreshold amplitude and phase resonance in models of quadratic type: Nonlinear effects generated by the interplay of resonant and amplifying currents. Journal of Computational Neuroscience 38(2):325-354, DOI 10.1007/s10827-014-0544-2.

Rotstein H, Oppermann T, White J, Kopell N (2006) The dynamic structure underlying subthreshold oscillatory activity and the onset of spikes in a model of medial entorhinal cortex stellate cells. Journal of Computational Neuroscience 21(3):271-292, DOI 10.1007/s10827-006-8096-8.

Rudolph M, Piwkowska Z, Badoual M, Bal T, Destexhe A (2004) A method to estimate synaptic conductances from membrane potential fluctuations 91(6):2884-2896, DOI 10.1152/jn.01223.2003.

Tao L, Shelley M, McLaughlin D, Shapley R (2004) An egalitarian network model for the emergence of simple and complex cells in visual cortex. Proceedings of the National Academy of Sciences of the United States of America 101(1):366-371, DOI 10.1073/pnas.2036460100.

Wang XJ (1998) Calcium coding and adaptive temporal computation in cortical pyramidal neurons. J Neurophysiol 79(3):1549-1566.

Wehr M, Zador AM (2003) Balanced inhibition underlies tuning and sharpens spike timing in auditory cortex. Nature 426(6965):442-446, DOI 10.1038/nature02116.

\section{Appendix A: Mathematical model of the pirami- dal cell}

The first model we use is the one given in Wang (1998), which describes the behaviour of a neuron that has two compartments, the dendrite and the soma plus the axonal initial segment. In our study we only consider the somatic compartment:

$$
C_{m} \frac{d V}{d t}=-I_{L}-I_{N a}-I_{K}-I_{C a}-I_{A H P}+I_{a p p}-I_{s y n},
$$


where $C_{m}$ is the capacitance, $I_{\text {syn }}$ the synaptic current, $I_{a p p}$ the applied current and $I_{L}, I_{i o n}$ the leak and the respective ion currents which are described by equations

$$
\begin{aligned}
& I_{L}=g_{L}\left(v-V_{L}\right), \\
& I_{N a}=g_{N a} m_{\infty}^{3}(v) h\left(v-V_{N a}\right), \\
& I_{K}=g_{K} n^{4}\left(v-V_{K}\right), \\
& I_{C a}=g_{C a} m l_{\infty}\left(v-V_{C a}\right), \\
& I_{A H P}=g_{A H P} \frac{c}{c+K_{D}}\left(v-V_{K}\right), \\
& I_{L T S}=g_{L T S} m_{L T S, \infty}^{3} h_{L T S}\left(v-V_{C a}\right),
\end{aligned}
$$

where $V_{i o n}$ and $g_{i o n}$ represent the specific ion reversal potentials and maximal conductances, respectively, $c$ is the intracellular calcium concentration $\left[\mathrm{Ca}^{2+}\right]$ and $K_{D}$ represents a growth factor of the $I_{A H P}$ current. The variables $h$ and $n$ are gating variables governed by first-order kinetics of type

$$
\dot{w}=\frac{d w}{d t}=\phi\left[\alpha_{w}(v)(1-w)-\beta_{w}(v) w\right]=\phi \frac{w_{\infty}(v)-w}{\tau_{w}(v)}
$$

The $m$-type variables are considered to be at the steady-state $m l=m l_{\infty}(v), m=$ $m_{\infty}(v)$. More precisely, the functions describing the gating dynamics are given by:

$$
\begin{aligned}
& w_{\infty}(v)=\alpha_{w}(v) /\left(\alpha_{w}(v)+\beta_{w}(v)\right), \\
& \tau_{w}(v)=1 /\left(\alpha_{w}(v)+\beta_{w}(v)\right), \\
& \alpha_{h}(v)=0.07 \exp (-(v+50) / 10), \\
& \beta_{h}(v)=1 /(1+\exp (-0.1(v+20))) \\
& \alpha_{n}(v)=-0.01(v+34) /(\exp (-0.1(v+34))-1), \\
& \beta_{n}(v)=0.125 \exp (-(v+44) / 25), \\
& \alpha_{m}(v)=-0.1(v+33) /(\exp (-0.1(v+33))-1), \\
& \beta_{m}(v)=4 \exp (-(v+58) / 12), \\
& m l_{\infty}(v)=1 /(1+\exp (-(v+20) / 5))
\end{aligned}
$$

and, for the LTS current,

$$
\begin{aligned}
& m_{L T S, \infty}(v)=1 /(1+\exp (-(v+65) / 7.8)), \\
& d h_{L T S} / d t=\left(\phi_{L T S}\left(h_{L T S, \infty}(v)-h_{L T S}(v)\right)\right) / \tau_{L T S}(v),
\end{aligned}
$$

where

$$
\begin{aligned}
& h_{L T S, \infty}(v)=1 /(1+\exp ((v+81) / 11)), \\
& \tau_{L T S}(v)=h_{L T S, \infty}(v) \exp ((v+162.3) / 17.8) .
\end{aligned}
$$

The intracellular calcium concentration $c=\left[\mathrm{Ca}^{2+}\right]$ is assumed to be governed by a leaky-integrator

$$
d c / d t=-\alpha I_{C a}-c / \tau_{C a},
$$

where $\tau_{C a}$ is the time constant and $\alpha$ is proportional to the membrane area divided by the volume below the membrane. 
The biophysical parameters are:

$$
\begin{gathered}
\text { Conductances }\left(\mathrm{mS} / \mathrm{cm}^{2}\right): g_{L}=0.1, g_{N a}=45, \\
g_{K}=18, g_{C a}=1.0, g_{A H P}=5.0, g_{L T S}=0.5 ; \\
\text { Reversal potentials }(m V): V_{L}=-65, V_{N a}=55, \\
\quad V_{K}=-80, V_{C a}=120.0, V_{E}=0, V_{I}=-80 ; \\
\text { Capacitance }\left(\mu F / \mathrm{cm}^{2}\right): C_{m}=1 ; \\
\text { Non - dimensional constants }: \phi=4, \phi_{L T S}=2 ; \\
\text { Other constants }: \alpha=0.002 \mu M(m s \mu A)^{-1} \mathrm{~cm}^{2}, \\
\quad \tau_{C a}=80 m s, K_{D}=30.0 \mu M .
\end{gathered}
$$

Finally, we take $I_{a p p} \in[-1,1] \mu \mathrm{A} / \mathrm{cm}^{2}$ (see Appendix B in Guillamon et al (2006) for a justification of this choice).

\section{Appendix B: Mathematical model of the stellate cell}

The second model we use is the one given in Rotstein et al (2006) by considering only the persistent sodium current $\left(I_{N a P}\right)$ and a fast-component h-current $\left(I_{h}\right)$, so the spiking currents are supposed inactivated. Except for those ionic currents, the remainder parameters of the model follow the same equations given in Appendix A. Then, the ionic currents are described as

$$
\begin{aligned}
& I_{N a P}=g_{p} p_{\infty}(v)\left(v-V_{N a}\right) \\
& I_{h}=g_{h} r_{f}(v)\left(v-V_{h}\right)
\end{aligned}
$$

where $g_{p}$ and $g_{h}$ are the maximal conductances, $V_{N a}$ and $V_{h}$ the reversal potentials, and $p_{\infty}$ and $r_{f}$ are the gating variables, all of them for the persistent sodium current and the h-current, respectively.

Note that the gating variable of $I_{N a P}$ has been approximated, since it is evolving a fast time scale, by the adiabatic approximation $p(v)=p_{\infty}(v)$. On the other hand, the gating variable $r_{f}$ is supposed to be governed, as in Appendix A, by first-order kinetics of type (12) where $\phi=1$. The functions defining $p_{\infty}$, $r_{f, \infty}$ and $\tau_{r_{f}}$ are, respectively,

$$
\begin{aligned}
& p_{\infty}(v)=1 /(1+\exp ((v+38) / 6.5)), \\
& r_{f, \infty}(v)=1 /(1+\exp ((v+79.2) / 9.78)), \\
& \left.\tau_{r_{f}}(v)=0.51 /(\exp ((v 1.7) / 10)+\exp ((v+340) / 52))\right)+1,
\end{aligned}
$$

The biophysical parameters through this model are:

$$
\begin{aligned}
& \text { Conductances }\left(\mathrm{mS} / \mathrm{cm}^{2}\right): g_{L}=0.5, g_{p}=0.5, g_{h}=1.5 \\
& \text { Reversal potentials }(m V): V_{L}=-65, V_{N a}=55 \\
& \quad V_{h}=-20, V_{E}=0, V_{I}=-80 \\
& \text { Capacitance }\left(\mu F / \mathrm{cm}^{2}\right): C_{m}=1 ; \\
& \text { Non - dimensional constants }: \phi=1
\end{aligned}
$$

Finally, we take $I_{a p p} \in[-4,-3] \mu A / \mathrm{cm}^{2}$. 


\section{Supplementary figures}

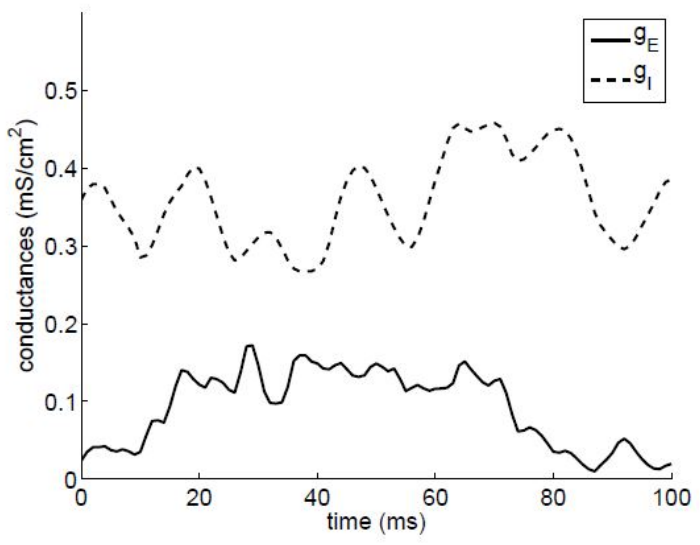

Supplementary Figure 1: Complete conductance traces. Complete $(1 \mathrm{~ms})$ time courses of the excitatory $\left(g_{E}(t)\right.$, solid trace) and inhibitory $\left(g_{I}(t)\right.$, dashed trace) conductances used to drive the activity of the target neuron. The conductance traces have been obtained from a computational network that models layer $4 \mathrm{C} \alpha$ of primary visual cortex (see McLaughlin et al (2000) and Tao et al (2004)). 